\title{
A J-band Transceiver MMIC with image rejection
}

\author{
C. A. Zelley, P. A. Gould*, P. D. Munday and R. W. Ashcroft \\ DERA, Malvern, Worcestershire, United Kingdom \\ *Bell Laboratories, Lucent Technologies, Swindon, UK. \\ Email: cazelley@dera.gov.uk
}

\begin{abstract}
This paper describes a J-band (15 GHz) transceiver MMIC with image rejection that has been designed for RADAR T/R module applications. The MMIC consists of a switched attenuator, an LNA, a Transmit/Receive switch, an image rejection mixer and a local oscillator buffer amplifier. The size of the MMIC is $6.48 \mathrm{~mm}$ by $4.47 \mathrm{~mm}$. In receive mode the MMIC has a conversion gain in excess of $10 \mathrm{~dB}$ with less than $1 \mathrm{~dB}$ of ripple and noise figure less than $4 \mathrm{~dB}$ across a $10 \mathrm{GHz}$ to $16.5 \mathrm{GHz}$ band. The Input P1dB is $-13 \mathrm{dBm}$. In transmit mode the conversion gain is $\mathbf{- 1 2} \mathrm{dB}$ with an input $\mathrm{P} 1 \mathrm{~dB}$ of +15 dBm. The Transceiver MMIC was fabricated using the GMMT, $0.25 \mu \mathrm{m}, \mathrm{H} 40$ pHEMT process. This is believed to be the first integrated transceiver MMIC designed specifically for this frequency band.
\end{abstract}

\section{INTRODUCTION}

There is an increasing interest in J-band frequencies for both communication and radar applications. At Jband module spacing in phased array applications dictates an integrated RF solution. Monolithic Microwave Integrated Circuits (MMIC) are an important technology for realising cost effective circuit functions in a small area. They offer increased reliability and increased uniformity between modules. As the technology matures there is a trend towards increased levels of functionality on the MMICs. This increased functionality is accomplished through greater integration. In this paper a J-band transceiver MMIC, which has a high level of functionality realised through the integration of a number of circuit blocks, is presented. Many previous transceiver MMICs have focused on the lower communication bands below 5 $\mathrm{GHz}$ [1], [2] and [3]. At higher frequencies a multi-chip module approach has been used, [4], [5]. Integrated transceivers MMIC have been presented [6], [7] and [8], for X-band and K-band applications. The transceiver MMIC presented here is believed to be the first integrated transceiver designed specifically for use at $\mathrm{J}$ band.

A description of the design and performance of the individual circuit blocks is given, followed by a description of the integrated transceiver. The design approach is described and the transceiver performance is presented. The integrated transceiver MMIC circuit consists of a switched attenuator, an LNA, a Transmit/Receive switch, an image rejection mixer and a LO buffer amplifier. A chip photograph and functional block diagram is given in Fig. 1 and Fig.2 respectively. An image rejection mixer is used so that the beam forming can be performed digitally.

\section{DESIGN AND MEASUREMENTS}

The transceiver chip photograph and configuration are presented in Fig. 1 and Fig. 2. The integrated transceiver MMIC circuit consists of a switched attenuator. The output of the switched attenuator forms the input to an LNA. The LNA is connected to an image rejection mixer via a Transmit/Receive switch. An image rejection mixer is used so that the beam forming functions can be performed digitally. The mixer is driven by an LO buffer amplifier. During transmit mode the switched attenuator is in an high attenuation state and the LNA is switched off, to reduce loop gain. The gate and drain bias lines for the different amplification stages of the LNA and LO buffer were combined and the control lines for the attenuator and switch were combined. This was done to reduce the number of DC bond pads on the chip, due to the problems of feeding the DC connections to the chip within the tight size constraints of the T/R module.

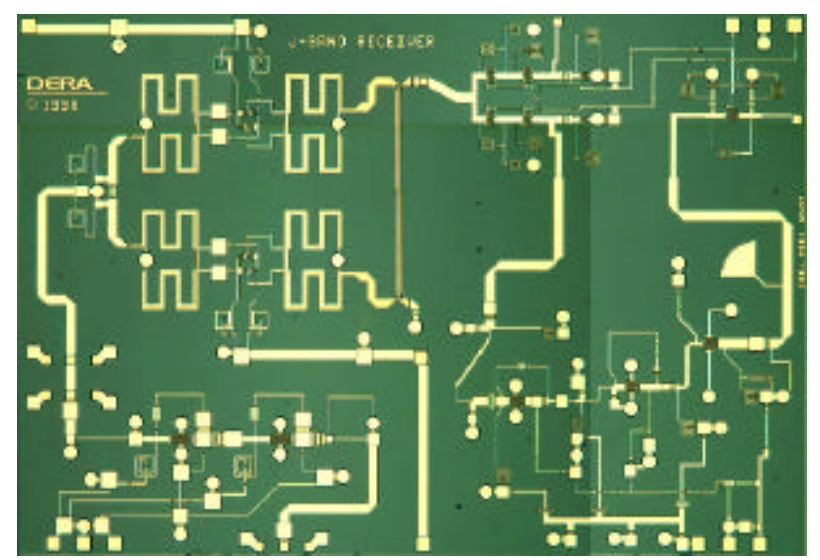

Figure 1, Photograph of the J-band transceiver MMIC 


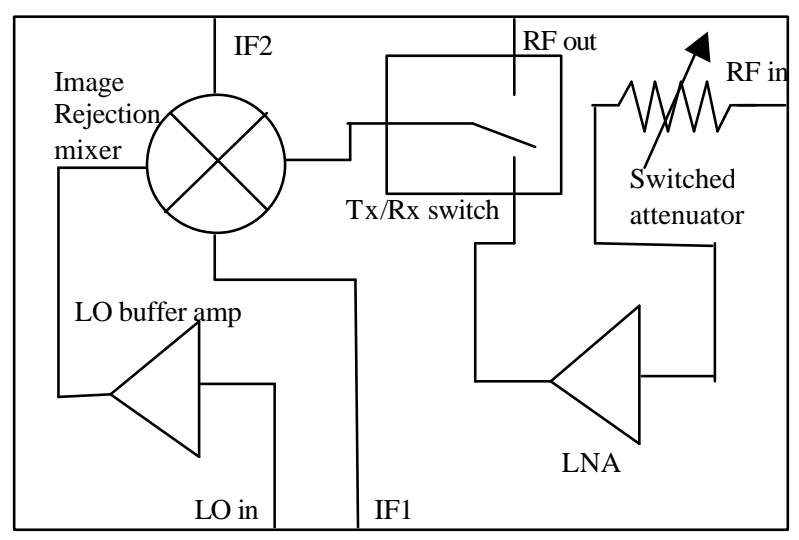

Figure 2, Block diagram of the J-band transceiver MMIC

The function of the switched attenuator is to protect the LNA amplifier and prevent feedback during Transmit mode. The attenuator contains three pHEMT devices arranged in a 'pi' configuration. Each transistor has an integrated spiral inductor connected across the source and drain terminals to resonate out the transistor parasitic capacitance. The shunt transistors are in series with $50 \mathrm{Ohm}$ resistors, which ensure that a good match is maintained during the high attenuation state. The return losses for the attenuator between $10 \mathrm{GHz}$ to 20 $\mathrm{GHz}$ is better than $10 \mathrm{~dB}$ in the 'on' state and better than $12 \mathrm{~dB}$ in the 'off' state. The 'off' state return loss between $13 \mathrm{GHz}$ and $17 \mathrm{GHz}$ was measured to be better than $20 \mathrm{~dB}$. The switched attenuator has an isolation of greater than $20 \mathrm{~dB}$ from $13 \mathrm{GHz}$ to $16.5 \mathrm{GHz}$ and greater than $15 \mathrm{~dB}$ from $12 \mathrm{GHz}$ to $18 \mathrm{GHz}$ in the 'off' state. In the 'on' state an insertion loss of $1.0 \mathrm{~dB}$ was observed from $13 \mathrm{GHz}$ to $18 \mathrm{GHz}$. The insertion loss at $12 \mathrm{GHz}$ was $1.5 \mathrm{~dB}$.

The Low Noise Amplifier has three stages and makes use of pHEMT transistors with a 0.25 um gate length. The first two transistor stages use source degeneration to simultaneously provide low noise and a good input port match. The second and third stages make use of parallel LR feedback for gain shaping and stability. The Noise Figure of the LNA is less than $3 \mathrm{~dB}$ with a gain in excess of $22 \mathrm{~dB}$ from $10 \mathrm{GHz}$ to $18 \mathrm{GHz}$. The LNA is biased using a single drain voltage of $3 \mathrm{~V}$ and a single gate voltage of $-0.46 \mathrm{~V}$. The bias current of the LNA circuit is approximately $160 \mathrm{~mA}$. The LNA was matched to operate in a $50 \mathrm{Ohm}$ environment. The input and output return loss was measured to be better than 15 dB from $12 \mathrm{GHz}$ to $19 \mathrm{GHz}$.

The transmit/receive switch is a Single Pole Double Throw (SPDT) absorptive switch. It connects both transmit and receive paths of the transceiver to the mixer circuit. Each path contains two pHEMT transistors in series and a single shunt device. The shunt transistors are in series with $50 \mathrm{Ohm}$ resistors so that a good return loss is maintained at all the switch ports, regardless of switch settings. The drain-source parasitic capacitance of the devices are resonated out using spiral inductors. The each half of the switch circuit has an insertion loss of less than $2.5 \mathrm{~dB}$ from $12 \mathrm{GHz}$ to 16 $\mathrm{GHz}$. The insertion loss at $18 \mathrm{GHz}$ is $2.7 \mathrm{~dB}$. The return losses for the low attenuation part of the switch are better the $10 \mathrm{~dB}$ from $12 \mathrm{GHz}$ to $20 \mathrm{GHz}$. The high attenuation path through the switch provides an isolation of $30 \mathrm{~dB}$ from $13 \mathrm{GHz}$ to $15 \mathrm{GHz}$ and an isolation of better than $26 \mathrm{~dB}$ between $12 \mathrm{GHz}$ and 18 $\mathrm{GHz}$. The return loss of the port connected to the common node via the high attenuation path is better than $17 \mathrm{~dB}$ from $12 \mathrm{GHz}$ to $18 \mathrm{GHz}$.

The Image rejection mixer consists of two doublebalanced mixers which are realised by configuring four 0.25 um gate length pHEMT transistors as diodes by connecting the source and drain together. The diode rings are fed with balanced signals. The balanced signals are obtained using Marchand baluns realised using edge coupled, meandered transmission line. The design of these balun structures is believed to be original. The two double-balanced mixers are used as in-phase and quadrature channels. A 90 degree $3 \mathrm{~dB}$ signal split is produced through the use of a Lange coupler at the RF portion of the mixer. The LO signal is fed to the two mixers using an in-phase lumped Wilkinson divider. The mixer has an in-phase and quadrature IF output which are combined using an offchip IF quadrature coupler. The Image rejection mixer has a conversion loss of $8 \mathrm{~dB}$ and image rejection of better than $20 \mathrm{~dB}$ from $12 \mathrm{GHz}$ to $18 \mathrm{GHz}$. The $\mathrm{LO}$ drive level required by the mixer is $15 \mathrm{dBm}$. The mixer ports are matched to $50 \mathrm{Ohms}$.

The LO buffer amplifier consists of two stages. Each stage contains a $0.25 \mathrm{um}$ gate length pHEMT transistor with parrallel LR feedback. The amplifier has a gain of greater than $12 \mathrm{~dB}$ from $12 \mathrm{GHz}$ to $18 \mathrm{GHz}$. The $\mathrm{LO}$ buffer amplifier is biased using a single drain supply voltage of $3.0 \mathrm{~V}$ and a single gate voltage of $-0.3 \mathrm{~V}$. The current consumption of the circuit is $240 \mathrm{~mA}$. The amplifier was matched to $50 \mathrm{Ohms}$. The input return loss was measured to be better than $15 \mathrm{~dB}$ from $6 \mathrm{GHz}$ to $17 \mathrm{GHz}$. At $18 \mathrm{GHz}$ the input return loss was better than $10 \mathrm{~dB}$. The output return loss was seen to be better than $15 \mathrm{~dB}$ from $12 \mathrm{GHz}$ to $20 \mathrm{GHz}$.

The circuits blocks described above were designed using HP Eesof, for electrical simulation, Sonnet, for electromagnetic simulations and were laid out using Barnard microsystems Wavemaker. Great care was taken during lay out to minimise electromagnetic coupling between adjacent components. Each circuit was simulated extensively to assess performance against temperature and process variations, prior to integration. Then extensive simulations were performed on the 
integrated circuit. A chip photograph of the integrated circuit is presented in Fig. 1.

\section{INTEGRATED MMIC MEASUREMENTS}

The integrated transceiver MMIC size is $6.48 \mathrm{~mm} \mathrm{x}$ $4.47 \mathrm{~mm}$. The MMIC is biased using a drain voltage of $3 \mathrm{~V}$, with a total required current of $400 \mathrm{~mA}$. The LO input port return loss was measured to be better than 10 $\mathrm{dB}$ from $10 \mathrm{GHz}$ to $17.5 \mathrm{GHz}$, as seen in Fig 3. The performance of the transceiver can be separated into two modes, receive mode and transmit mode.

\section{S11 FORWARD REFLECTION}

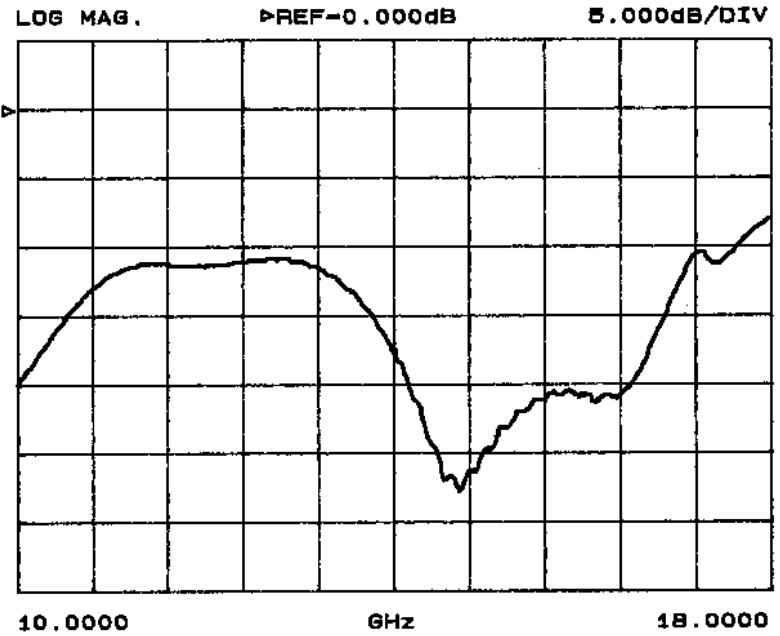

Figure 3, LO input port return loss

\section{A. Receive mode}

The overall Noise Figure of the MMIC was less than $4 \mathrm{~dB}$ with an associated conversion gain greater than 10 $\mathrm{dB}$ with less than $1 \mathrm{~dB}$ ripple (see Fig. 4). The LO drive level required by the chip is $+4 \mathrm{dBm}$. The level of image rejection achieved is better than $20 \mathrm{~dB}$ (using an external quadrature coupler). The LO to IF isolation was measured to be $48.17 \mathrm{~dB}$ and the RX to IF isolation was measured to be $32.0 \mathrm{~dB}$, at $15 \mathrm{GHz}$. The Input $1 \mathrm{~dB}$ compression point was measured to be $-13 \mathrm{dBm}$.

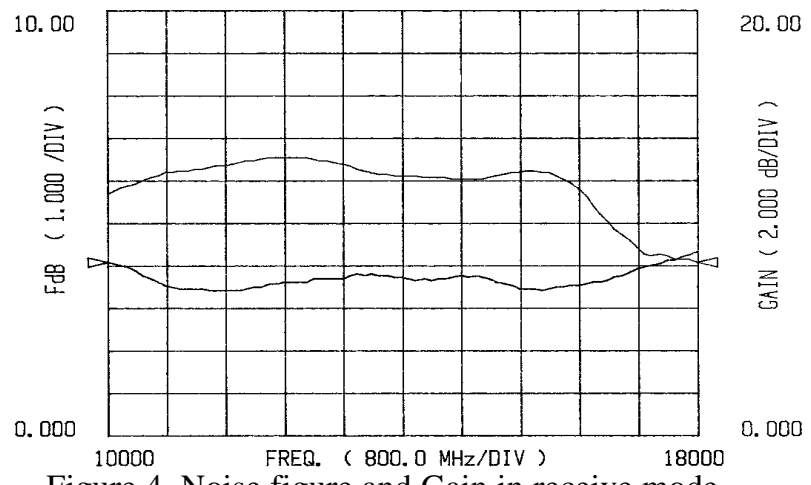

Figure 4, Noise figure and Gain in receive mode
In receive mode the return losses of the transmit and receive ports were measured to be better than $7 \mathrm{~dB}$ across the band, as seen in Fig. 5 and Fig. 6. The Rx port match was worse than expected at approximately $15 \mathrm{GHz}$. This is believed to be due to the chip numbering text that was placed close to the LNA input line prior to mask fabrication. The port match measurements of the individual LNA block gave excellent agreement with simulations.

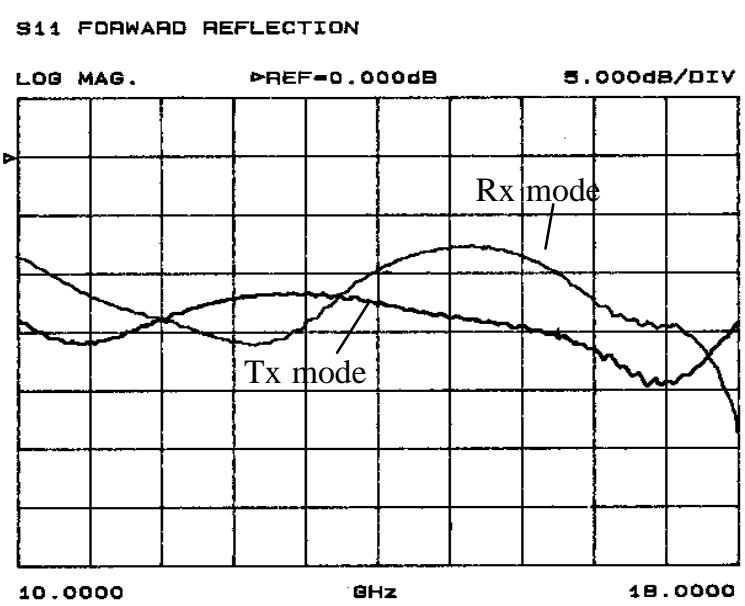

Figure 5, Receive port return loss

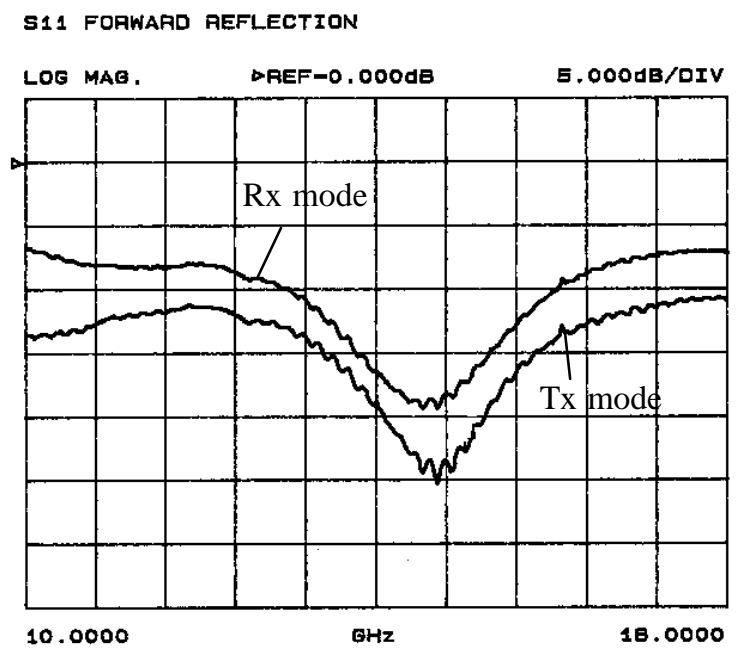

Figure 6, Transmit port return loss

\section{B. Transmit mode}

The insertion loss of the transceiver in transmitter mode is $-12 \mathrm{~dB}$. The image rejection achieved is better than $20 \mathrm{~dB}$ (using an external coupler). The LO to TX isolation was seen to be $36.67 \mathrm{~dB}$, at $15 \mathrm{GHz}$. The $\mathrm{Rx}$ to Tx isolation at $15 \mathrm{GHz}$ was $41.45 \mathrm{~dB}$ with the LNA switched off. In transmit mode the input $1 \mathrm{~dB}$ compression point was measured to be $+15 \mathrm{dBm}$. In 
transmit mode the return loss of the transmit and receive ports was better than $10 \mathrm{~dB}$ across the band, as seen in Fig. 5 and Fig. 6.

\section{CONCLUSION}

In this paper a J-band transceiver MMIC with image rejection has been described. The MMIC consists of a switched attenuator, a low noise amplifier (LNA), a Transmit/Receive switch, an image rejection mixer and a local oscillator buffer amplifier. The size of the MMIC is $6.48 \mathrm{~mm}$ by $4.47 \mathrm{~mm}$. In receive mode the MMIC has a conversion gain in excess of $10 \mathrm{~dB}$ and an associated noise figure less than $4 \mathrm{~dB}$ across a $10 \mathrm{GHz}$ to $17 \mathrm{GHz}$ band. The level of image rejection achieved was seen to be better than $20 \mathrm{~dB}$. The Transceiver MMIC was fabricated using the GMMT, $0.25 \mu \mathrm{m}$ gate length, H40 pHEMT process. The circuit contained a high level of integration, which was demonstrated to give excellent performance. The MMIC described is suitable for RADAR T/R module applications in the $10 \mathrm{GHz}$ to 17 $\mathrm{GHz}$ band. This is believed to be the first integrated transceiver MMIC designed specifically for this frequency band.

\section{ACKNOWLEDGEMENTS}

The authors gratefully acknowledge the contributions of Mark Moore, Geoff Ball, Andy Wicks and Greg Ball.

\section{REFERENCES}

[1] T. Quach, F. Bonn, J. Ortiz, M. Thomas, "A highly integrated commercial GaAs transceiver MMIC for $2.45 \mathrm{GHz}$ ISM applications", pp 141 IEEE WCC, 1997.

[2] J. Komiak, A. Agrawal, "Octave S/C band MMIC T/R modules for multifunction phased arrays", pp. 35, IEEE MMWMC-S, 1991.

[3] H. Ma, et al.,"A single chip $1.9 \mathrm{GHz}$ RF transceiver MMIC using GaAs MESFET technology", pp. 101, IEEE RFIC-S, 1998.

[4] M. Kanno, et al., "Miniturised X-band MMIC T/R module for conformal array antenna", pp. 17, IEEE MTT-S, 1996.

[5] J. Bugeau, W. Coughlin, M. Priolo, G. Onge", Advanced MMIC T/R module for 6 to $18 \mathrm{GHz}$ multifunction arrays pp. 119, IEEE MMWMC-S, 1992.

[6] I. Toyoda et al., "X-band Si Bipolar transistor single chip transceiver using three-dimensional MMIC technology" pp. 337, IEEE MTT-S, 1998.

[7] T. Sakia, N. Takeuchi, A. Mizobuchi, Y. Iyama, N. Tanino, "Ultra small size $\mathrm{X}$ band MMIC T/R module for active phased array", pp. 1531, IEEE MTT-S, 1992.

[8] H. Fudem, S. Moghe, G. Dietz, S. Consolazio, "A highly integrated MMIC K-band transmit/receive chip", pp. 119, IEEE MMWMC-S, 1993. 